\title{
Adsorption du cadmium dans les sols calcaires du Sud-Est de l'Espagne
}

\author{
H Halen, M Garcia-Navarro et R Van Bladel * \\ Université catholique de Louvain, Faculté des sciences agronomiques, unité des sciences du sol, \\ Place Croix du Sud, 2, B-1348 Louvain-la-Neuve, Belgique
}

(Reçu le 23 février 1990; accepté le 11 novembre 1990)

\begin{abstract}
Résumé - Les horizons de surface d'une série de 12 sols calcaires de la région du Sud-Est de l'Espagne sont comparés selon leur capacité de rétention du cadmium. Les résultats expérimentaux sont bien représentés par la relation de Freundlich et mettent en évidence 2 mécanismes distincts d'adsorption selon que les apports en Cd ont lieu à raison de quantités traces ou de quantités plus importantes. Dans les 2 cas, l'analyse statistique indique que les quantités de Cd adsorbées peuvent être valablement estimées à partir des valeurs de CEC $\left(\mathrm{ACNH}_{4}\right)$ et des teneurs en calcaire actif. La plus forte adsorption du Cd, par rapport aux sols non calcaires, s'explique par la réactivité des surfaces de calcite (chimisorption et nucléation-précipitation, respectivement aux taux d'apports faibles et élevés en Cd) ainsi que par les $\mathrm{pH}$ généralement élevés des sols étudiés $(7,6<\mathrm{pH}<8,6)$.

La comparaison des isothermes d'adsorption mesurées en milieu chlorure $\left(\mathrm{CaCl}_{2} 0,01 \mathrm{~mol} / \mathrm{l}^{-1}\right)$ et en milieu perchlorate $\left(\mathrm{Ca}\left(\mathrm{ClO}_{4}\right)_{2} \quad 0,01\right.$ mol.-1-1) montre que la rétention du Cd est principalement déterminée par l'activité du Cd²+ libre dans la solution du sol.
\end{abstract}

métal lourd / cadmium / sol calcaire / calcaire actif / adsorption / Espagne

Summary - Adsorption capacity of cadmium in some calcareous soils of South-Eastern Spain. Top-soil horizons of a series of 12 calcareous soils in South-Eastern Spain (table I) have been compared for their cadmium adsorption capacity. Data closely obey the Freundlich equation and indicate 2 distinct mechanisms of adsorption (fig 1 , table II) according to whether the cadmium in the bulk solution is present in trace or in higher amounts. In addition, some precipitation with a concomitant decrease in $\mathrm{pH}$ could also occur at initial concentrations of Cd higher than 5 $\mathrm{mg} / \mathrm{I}$ (fig 2). Statistical analysis (tables III and IV) points out that the adsorbed amounts of Cd may be estimated from the CEC $\left(\mathrm{AcNH}_{4}\right)$ values and the active calcium carbonate content. The agreement between the predictive values and the experimental ones is shown to be fairly good (fig 3). The higher adsorption of Cd in comparison with the noncalcareous soils is due to the reactivity of calcite surfaces (chemisorption and nucleation-precipitation, respectively at low and high rates of $\mathrm{Cd}$ ) and also to the commonly high $\mathrm{pH}$ of the soils studied $(7.6<\mathrm{pH}<8.6)$. Comparison of the adsorption isotherms measured in chloride $\left(\mathrm{CaCl}_{2} 0,01 \mathrm{~mol}^{-1}\right)$ and perchlorate $\left(\mathrm{Ca}\left(\mathrm{ClO}_{4}\right)_{2} 0,01 \mathrm{~mol}^{-1}\right)^{-1}$ media (fig 4) points out that adsorption of Cd is mainly determined by the activity of free $\mathrm{Cd}^{2+}$ in the soil solution.

heavy metal / cadmium / calcareous soil / active $\mathrm{CaCO}_{3}$ / adsorption / Spain 


\section{INTRODUCTION}

Avec l'industrialisation, le sol, outre son rôle premier de support à la production agricole et sylvicole, est de plus en plus amené à répondre à une seconde fonction : celle de milieu tampon pour l'environnement, face aux apports de substances polluantes.

Parmi ces substances polluantes, les métaux lourds occupent une place prioritaire. Ceux-ci sont ajoutés au sol, soit par l'intermédiaire des "additifs" agricoles habituels tels que engrais, lisiers, herbicides et fongicides, eaux d'irrigation, soit via l'apport de matériaux potentiellement nuisibles tels que boues d'épuration, composts urbains, "déchets miniers», aérosols industriels. Parmi les causes premières d'une concentration excessive de cadmium dans les sols, on peut citer la métallurgie du zinc ainsi que l'utilisation d'engrais superphosphates.

Du point de vue toxicologique, le Cd revêt un intérêt particulier étant donné son degré de toxicité élevé vis-à-vis des organismes biologiques en général, et son association avec un certain nombre de maladies humaines (Friberg et al, 1974; Kjellstrom et Nordberg, 1978; Roels et al, 1981; Ryan et al, 1982).

Ceci justifie l'importance des recherches effectuées ces dernières années dans le but de cerner les facteurs qui, dans les sols, contrôlent les processus de fixation ou de mobilisation du $\mathrm{Cd}$ : les risques de lessivage vers la nappe, tout comme ceux liés à l'absorption du métal par la plante (Mahler et al, 1980; Gerritse et al, 1983; Verloo et al, 1987), sont, en effet, déterminés par l'importance relative de la fraction soluble du métal dans le sol.

Généralement, la distribution du Cd entre les phases solide et liquide du sol est contrôlée par des processus d'adsorption (Alloway, 1990). Dans les terres non calcaires, le pouvoir de rétention $\mathrm{du} \mathrm{Cd}$ a été mis en relation avec certaines caractéristiques analytiques des sols, telles que le $\mathrm{pH}$, la capacité d'échange cationique CEC, le calcium échangeable ou la somme des bases échangeables (Singh, 1979; Mc Bride et al, 1981; King, 1988; Halen et Van Bladel, 1990), la teneur en matière organique (LéviMinzi et al, 1976) ainsi que le pH (Singh, 1979) et la teneur en $\mathrm{Fe}_{2} \mathrm{O}_{3}$ (Garcia-Miragaya et Page, 1978).

Dans les sols calcaires, le comportement du $\mathrm{Cd}$ est susceptible d'être influencé, dans une certaine mesure, par la présence des minéraux carbonatés, lesquels présentent des surfaces adsorbantes potentiellement importantes pour les métaux lourds (Leeper, 1952; Alloway et al, 1988). Vis-à-vis du cadmium, la grande affinité des surfaces de $\mathrm{CaCO}_{3}$ aux faibles concentrations en $\mathrm{Cd}$ s'explique par des réactions de chimisorption attribuées à la similarité entre les rayons ioniques du $\mathrm{Cd}^{2+}$ et du $\mathrm{Ca}^{2+}$ ( $\mathrm{Mc}$ Bride, 1980; Papadopoulos et Rowell, 1988). Cependant, aux concentrations plus élevées, l'activité du $\mathrm{Cd}^{2+}$ en solution peut être contrôlée par la précipitation de $\mathrm{CdCO}_{3}$ (Street et al, 1977; Cavallaro et Mc Bride, 1978; Soon, 1981).

Parmi les différentes pratiques agronomiques généralement proposées pour limiter les problèmes d'environnement liés à la mobilisation des métaux lourds dans les écosystèmes, la sélection de types de sol apparaît comme une possibilité de premier plan. Etant donné la forte réactivité du $\mathrm{CaCO}_{3}$ vis-à-vis des métaux lourds et en particulier, vis-à-vis du cadmium, le caractère plus ou moins calcaire des sols pourrait être un élément à prendre en considération dans le choix des terres susceptibles de recevoir des additifs chargés en métaux lourds.

Cependant, dans le cas des sols calcaires, peu de travaux ont été entrepris dans le but de relier la capacité de rétention du Cd aux propriétés physico-chimiques générales du sol. En particulier, la relation entre le pouvoir d'adsorption du $\mathrm{Cd}$ et la teneur du sol en $\mathrm{CaCO}_{3}$ n'a pu encore être établie clairement. Elle ressort notamment de l'étude publiée par Estañ et al (1987) mais ne transparaît pas dans les travaux de Lévi-Minzi et al (1976), Navrot et al (1978) et O'Connor et al (1984).

C'est l'objet de cet article, où l'on compare les horizons de surface d'une série de 12 sols calcaires de la région du Sud-Est de l'Espagne, selon leur capacité à fixer le $\mathrm{Cd}$.

\section{MATÉRIAUX ET MÉTHODES}

\section{Sols}

Les échantillons de terre des 12 horizons de surface sélectionnés pour cette étude proviennent des provinces de Murcie et de Valence, dans le Sud-Est de l'Espagne.

Ils diffèrent sensiblement entre eux quant à leur texture et leur teneur en calcaire, mais sont relativement homogènes quant à leur $\mathrm{pH}(7,6-8,6)$. Trois des sols étudiés ont un caractère salin (sols 3,4 et 9 ) et 3 autres (sols 6,10 et 12) sont salins sodiques (ESP > $15 \%)$. 
Les principales propriétés physico-chimiques des échantillons de terre séchés à l'air et tamisés à $2 \mathrm{~mm}$ sont renseignées au tableau I. La granulométrie a été réalisée d'après la méthode de la sédimentation, sans destruction du $\mathrm{CaCO}_{3}$, et la minéralogie par diffractométrie aux rayons $X$. Le $\mathrm{pH}$ a été mesuré pour un rapport sol/solution de 1/2.

Le $\mathrm{CaCO}_{3}$ équivalent total a été calculé après neutralisation acide (Allison et Moodie, 1965) et le calcaire actif après extraction à l'oxalate d'ammonium $0,2 \mathrm{~N}$ selon la méthode Drouineau (Bonneau et Souchier, 1979).

Les teneurs totales en fer et en manganèse, après digestion des sols à l'acide fluorhydrique, et celle du $\mathrm{Fe}_{2} \mathrm{O}_{3}$ libre, après extraction au dithionite de sodium d'après la méthode Dithionite Citrate Bicarbonate, ont été déterminées par spectrophotométrie d'absorption atomique. La capacité d'échange cationique (CEC) a été mesurée par la méthode à l'acétate d'ammonium $\mathrm{N}$ à $\mathrm{pH} 7$ ainsi que par la méthode de la dilution isotopique (Van Bladel et Menzel, 1969) au moyen du radioisotope ${ }^{45} \mathrm{Ca}$.

Les cations échangeables $\mathrm{Na}^{+}$et $\mathrm{K}^{+}$ont été analysés par absorption atomique après extraction à l'acétate d'ammonium $\mathrm{N}$ à $\mathrm{pH} 7$. La teneur en $\left(\mathrm{Ca}^{2+}+\right.$ $\mathrm{Mg}^{2+}$ ) a été calculée par différence avec la valeur de la $\mathrm{CEC}\left(\mathrm{AcNH}_{4}\right)$.

\section{Isothermes d'adsorption}

Les isothermes d'adsorption ont été réalisées selon la technique de mise en équilibre par agitation, en utilisant une solution saline $\left(\mathrm{CaCl}_{2}, 0,01 \mathrm{~mol} \bullet-1\right)$ de concentrations différentes en chlorure de cadmium $(0,01,0,1,0,5,1,5,10,20,100$ et $1000 \mathrm{mg} / \mathrm{l} \mathrm{Cd})$.

Les chlorures ont été préférés aux nitrates et aux perchlorates parce que le chlorure est une composante essentielle des sols à caractère salin. Quelques expériences ont cependant été réalisées dans une solution de perchlorate afin d'étudier l'influence de l'anion complémentaire. Après marquage des solutions initiales au ${ }^{109} \mathrm{Cd}(\gamma=0,088 \mathrm{MeV}, T=453 \mathrm{j}), 25 \mathrm{ml}$ de chaque solution sont ajoutés dans des tubes en polyéthylène contenant $0,4 \mathrm{~g}$ de sol. Après agitation dans une enceinte thermostatée à $20^{\circ} \mathrm{C}$ pendant $16 \mathrm{~h}$, l'équilibre étant atteint, les suspensions sont centrifugées à $15000 \mathrm{~g}$. On mesure le $\mathrm{pH}$ et la conductivité électrique du surnageant et on prélève une aliquote de $5 \mathrm{ml}$ dont l'activité est enregistrée par un spectromètre $\gamma$ Canberra. Les quantités adsorbées de cadmium se calculent par différence entre les concentrations initiales et d'équilibre.

Les données d'adsorption sont traitées suivant l'équation linéarisée de Freundlich :

$$
\log Q=\log K_{F}+n \log C(\text { ou } A)
$$

où $Q$ représente la quantité de $C d$ adsorbée par unité de poids de sol (mg/kg); $C$ (ou $A$ ), la concentration totale en $\mathrm{Cd}$ (ou l'activité en $\mathrm{Cd}^{2+}$ ) dans la solution d'équilibre $(\mathrm{mg} / \mathrm{l}) ; K_{\mathrm{F}}$, le coefficient de Freundlich qui représente la quantité de $\mathrm{Cd}$ adsorbée pour une concentration (ou activité) unitaire de métal lourd dans la solution d'équilibre $\left(\mathrm{mg} \cdot \mathrm{kg}^{-1} / \mathrm{mg}^{\circ} \mathrm{I}^{-1}\right)$ et , $n$, le paramètre d'intensité de la réaction d'adsorption reflétant le degré de non-linéarité de l'isotherme. Les valeurs de $K_{F}$ et $n$ se calculent par régression linéaire entre les valeurs expérimentales de $\log Q$ et $\log C$.

\section{RÉSULTATS}

\section{Isothermes d'adsorption}

Quelques isothermes d'adsorption représentatives, traitées selon le modèle de Freundlich, sont données sur la figure 1 . On constate que les points expérimentaux se répartissent selon 2 segments de droites aux pentes distinctes. Aux faibles taux d'apport en Cd, correspondant aux concentrations initiales de $0,01,0,1,0,5,1$ et $5 \mathrm{mg} / \mathrm{Cd}$, la pente est légèrement inférieure à l'unité (segment 1). Pour les concentrations initiales supérieures à $5 \mathrm{mg} / \mathrm{l} \mathrm{Cd}$ (segment 2), l'augmentation d'adsorption est proportionnellement moindre, ce qui se traduit par une plus faible valeur de pente.

On peut aussi remarquer que la concentration en $\mathrm{Cd}$, à laquelle correspond le point d'intersection des 2 droites, semble être d'autant plus faible que la capacité d'adsorption des sols est élevée. Des isothermes de configuration similaire ont été observées par plusieurs auteurs (Street et al, 1977; Singh, 1979; Jarvis et Jones, 1980; Alloway et al, 1988) et notamment dans le cas de sols calcaires (O'Connor et al, 1984) ou de matériaux parentaux de sols calcaires, de même que pour l'adsorption du Cd par de la cal-

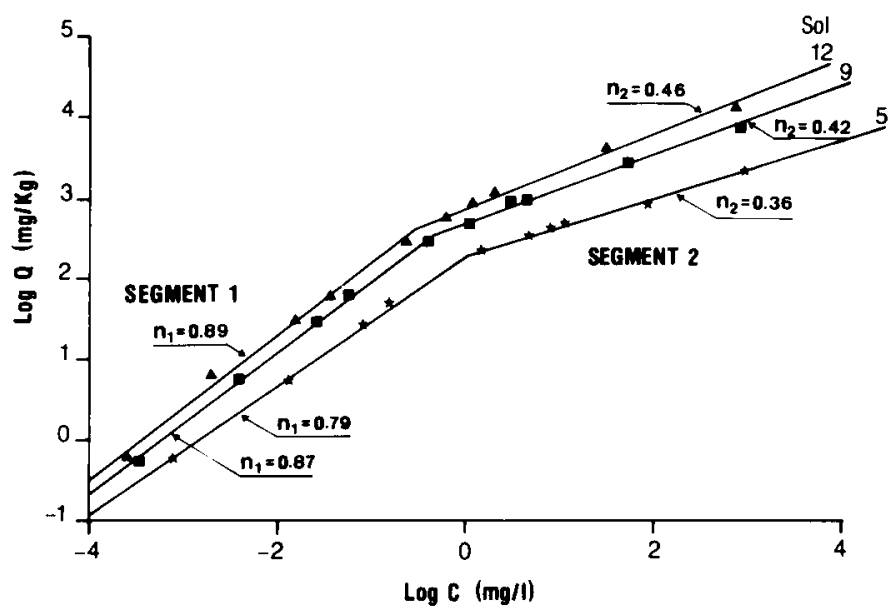

Fig 1. Application de la relation de Freundlich aux isothermes d'adsorption obtenues pour les sols 5,9 et 12 (tableau I). 


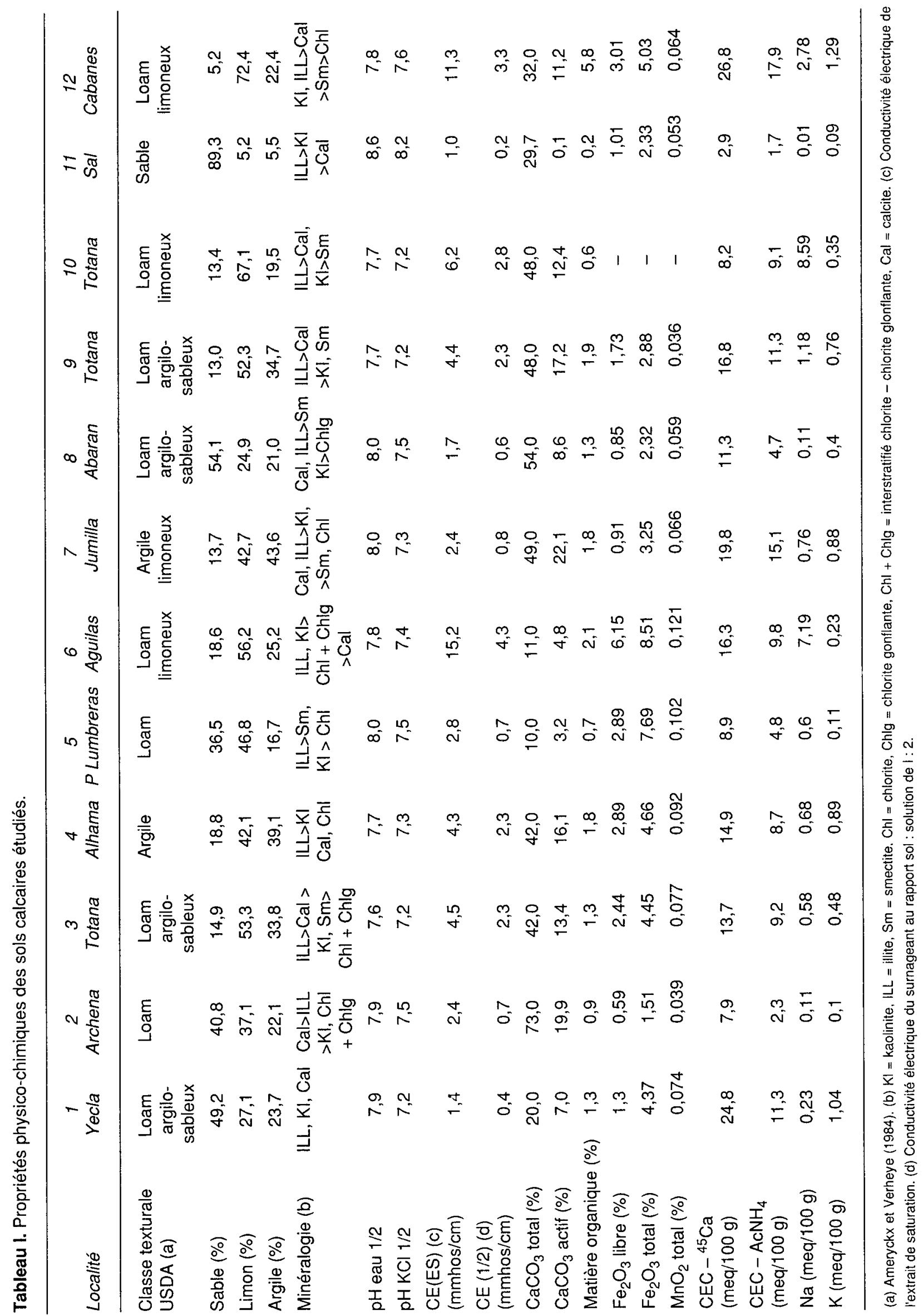


cite pure (Papadopoulos et Rowell, 1988). L'aspect brisé de l'isotherme est généralement attribué à l'existence de (au moins) 2 mécanismes distincts d'adsorption.

Les activités des ions libres $\mathrm{Cd}^{2+}$ ont pu être calculées, à la température de $25^{\circ} \mathrm{C}$, d'après les concentrations totales en cadmium et en chlorure, les constantes d'association $\left(\mathrm{K}^{\circ}\right)$ des paires ioniques $\mathrm{CdCl}^{+}, \mathrm{CdCl}_{2}{ }^{\circ}$ et $\mathrm{CdOH}^{+}$soit $10^{1,98}$, $10^{2,60}$ et $10^{-10,1}$ respectivement (Wagman et al, 1968; Street et al, 1977) et en considérant l'équation de Davies pour le calcul des coefficients individuels d'activités *. Ces activités ont été exprimées en fonction du $\mathrm{pH}$, comme illustré à la figure 2, ce qui permet de faire apparaître que, dans la première partie de l'isotherme, les solutions sont sous-saturées vis-à-vis du $\mathrm{CdCO}_{3}$ et le $\mathrm{pH}$ reste plus ou moins constant. Ce résultat est conforme à l'hypothèse d'une adsorption du cadmium par chimisorption. Dans la seconde partie de l'isotherme, les solutions d'équilibre sont généralement sursaturées en $\mathrm{CdCO}_{3}$. Une précipitation peut théoriquement avoir lieu, et a fortiori si l'on considère que la pression partielle en $\mathrm{CO}_{2}$ dans les suspensions a pu être supérieure à celle en équilibre avec l'atmosphère. Dans certains cas cependant (exemple: sol 12), la sursaturation n'est obtenue qu'avec les taux d'apports les plus élevés. Dans tous les cas, la précipitation se traduit par une diminution du $\mathrm{pH}$. Celle-ci peut être attribuée (Mc Bride, 1980; Papadopoulos et Rowell, 1988) à la réaction de précipitation $\mathrm{du} \mathrm{CdCO}_{3}\left(\mathrm{Cd}^{2+}+\mathrm{HCO}_{3}^{-} \rightleftharpoons \mathrm{CdCO}_{3}(\mathrm{~s})+\mathrm{H}^{+}\right)$ et au recouvrement concomittant des surfaces de calcite par le $\mathrm{CdCO}_{3}$. Ce recouvrement empê-

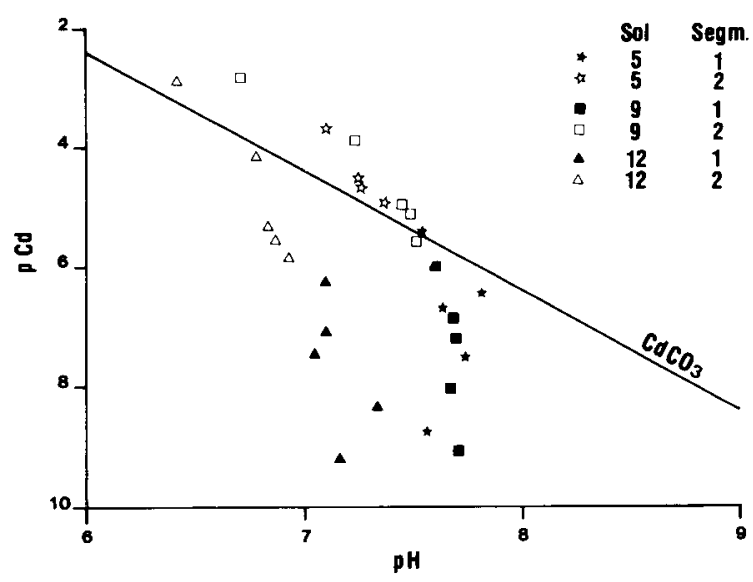

Fig 2. Diagramme de solubilité $(\mathrm{pCd}-f(\mathrm{pH}))$ pour les équilibres $\mathrm{Cd}^{2+}-$ sol et droite de solubilité du $\mathrm{CdCO}_{3}$ (pour $\mathrm{PCO}_{2}$ supposé égal à $32,68 \mathrm{~Pa}$ ). cherait le système $\mathrm{CaCO}_{3}-\mathrm{CO}_{2}-\mathrm{H}_{2} \mathrm{O}$ d'exercer son rôle de tampon vis-à-vis de l'acidité générée avec la formation du précipité.

\section{Quantification de l'adsorption et traitement statistique}

Les paramètres des régressions de Freundlich, calculés pour les segments 1 et 2 des isothermes sont repris au tableau II. Le paramètre $\mathrm{Q}_{10 \mathrm{ppb}}$, qui représente la quantité de $\mathrm{Cd}$ adsorbée pour une concentration de $0,01 \mathrm{mg} / \mathrm{l} \mathrm{Cd}$ dans la solution d'équilibre, est choisi comme indice représentatif de la capacité d'adsorption dans la zone des traces : il se calcule par régression sur les points expérimentaux correspondant aux concentrations initiales de 0,01-5 $\mathrm{mg} / \mathrm{l}$ (segment 1). Quant au paramètre $Q_{1000}$ $\mathrm{ppb}$, défini par analogie avec $Q_{10} \mathrm{ppb}$, pour une concentration d'équilibre de $1 \mathrm{mg} / \mathrm{l}$, il est retenu comme indice pour évaluer la capacité d'adsorption dans la zone des plus fortes concentrations en $\mathrm{Cd}$. Ce dernier paramètre se calcule par régression sur les points expérimentaux correspondant au segment 1 ou segment 2 des isothermes (concentrations initiales : 5 à $1000 \mathrm{mg} / \mathrm{l}$ ) selon que le point d'intersection des 2 droites se situe respectivement à droite (sols $5,8,10$ et 11) ou à gauche (cas général) du point $\log C=0$ (fig 1). II faut souligner qu'en valeur, le terme $\mathrm{Q}_{1000 \mathrm{ppb}}$ équivaut au coefficient $K_{F}$ dégagé des relations de Freundlich pour les segments $1\left(K_{1}\right)$ ou $2\left(K_{2}\right)$ des isothermes.

Une étude statistique a été réalisée afin de relier la capacité d'adsorption aux propriétés physico-chimiques des sols. Le sol 10 n'a pas été repris dans cette analyse, étant donné une augmentation sensible de la conductivité électrique, et donc également de la force ionique, dans les solutions équilibrées avec ce sol, ce qui vraisemblablement est à mettre en relation avec une dissolution de gypse.

Au tableau III figurent les coefficients de corrélation simple entre les paramètres $Q_{10}$ ppb et $\mathrm{Q}_{1000 \text { ppb }}$ et chaque propriété des sols. Les variables explicatives les mieux corrélées avec l'adsorption, tant dans la région des traces que dans celle des hautes concentrations, sont le calcaire actif, la teneur en argile, la $C E C$ $\left(\mathrm{ACNH}_{4}\right)$, la $C E C\left({ }^{45} \mathrm{Ca}\right)$ et la teneur en $(\mathrm{Ca}+$

\footnotetext{
* La contribution de l'espèce $\mathrm{CdCO}_{3}{ }^{\circ}\left(\mathrm{K}^{\circ}=10^{4,02}\right.$, Gardiner, 1974) peut être négligée dans la plage de pH considérée dans ce travail.
} 
Tableau II. Paramètres de Freundlich décrivant l'adsorption du cadmium pour 12 sols calcaires du sud-est de l'Espagne.

\begin{tabular}{|c|c|c|c|c|c|c|c|c|c|c|}
\hline \multirow{2}{*}{$\begin{array}{l}\text { Sol } \\
N^{\circ}\end{array}$} & \multicolumn{3}{|c|}{ Segment 1} & \multicolumn{3}{|c|}{ Segment 2} & \multirow{2}{*}{$\begin{array}{l}\mathrm{Q}_{10 p p b^{* *}} \\
(\mathrm{mg} / \mathrm{kg})\end{array}$} & \multirow{2}{*}{$\begin{array}{l}\text { Erreur sur } \\
\mathrm{Q}_{10 \text { ppb }} \\
(\%)\end{array}$} & \multirow{2}{*}{$\begin{array}{c}Q_{1000 p p b^{* *}} \\
(\mathrm{mg} / \mathrm{kg})\end{array}$} & \multirow{2}{*}{$\begin{array}{c}\text { Erreur sut } \\
Q_{1000 p p b} \\
(\%)\end{array}$} \\
\hline & $\begin{array}{c}\mathrm{K}_{1} \\
(\mathrm{mg} / \mathrm{kg})\end{array}$ & $n_{1}$ & $r_{1}^{*}$ & $\begin{array}{c}\mathrm{K}_{2} \\
(\mathrm{mg} / \mathrm{kg})\end{array}$ & $\mathrm{n}_{2}$ & $r_{2}^{*}$ & & & & \\
\hline 1 & 752,3 & 0,86 & 0,998 & 563,0 & 0,39 & 0,993 & 14,3 & 8,2 & 563,0 & 0,2 \\
\hline 2 & 1017,0 & 0,90 & 0,998 & 653,1 & 0,31 & 0,965 & 15,7 & 7,4 & 653,1 & 0,2 \\
\hline 3 & 523,7 & 0,84 & 0,999 & 474,7 & 0,43 & 0,985 & 11,0 & 10,1 & 474,7 & 0,2 \\
\hline 4 & 1040,2 & 0,87 & 0,999 & 660,4 & 0,36 & 0,983 & 18,8 & 5,9 & 660,4 & 0,2 \\
\hline 5 & 179,2 & 0,79 & 0,997 & 186,7 & 0,36 & 0,997 & 4,7 & 23,0 & 179,2 & 0,6 \\
\hline 6 & 481,8 & 0,80 & 0,998 & 385,9 & 0,41 & 0,998 & 12,1 & 9,8 & 385,9 & 0,3 \\
\hline 7 & 2632,2 & 0,91 & 0,999 & 1005,7 & 0,36 & 0,980 & 39,7 & 2,7 & 1005,7 & 0,1 \\
\hline 8 & 218,9 & 0,81 & 0,998 & 220,8 & 0,40 & 0,999 & 5,2 & 22,6 & 218,9 & 0,5 \\
\hline 9 & 671,2 & 0,87 & 0,999 & 472,3 & 0,42 & 0,994 & 12,2 & 9,4 & 472,3 & 0,2 \\
\hline 10 & 252,1 & 0,86 & 0,999 & 259,9 & 0,42 & 0,990 & 4,8 & 23,7 & 252,1 & 0,4 \\
\hline 11 & 142,6 & 0,73 & 0,996 & 146,4 & 0,43 & 0,999 & 5,0 & 25,1 & 142,6 & 0,9 \\
\hline 12 & 1166,9 & 0,89 & 0,996 & 720,9 & 0,46 & 0,993 & 19,3 & 6,5 & 720,9 & 0,1 \\
\hline \multicolumn{4}{|c|}{ Moyenne } & & & & 13,6 & & 474,4 & \\
\hline \multicolumn{4}{|c|}{ Ecart type } & & & & 9,8 & & 258,9 & \\
\hline \multicolumn{4}{|c|}{ Coefficient de variation } & & & & 72 & & 54 & \\
\hline
\end{tabular}

- Coefficient de corrélation pour la relation $\log Q$ vs $\log C_{1}{ }^{* *}$ Quantité de Cd adsorbée $\left(\mathrm{mg}^{*} \mathrm{~kg}^{-1}\right)$ correspondant à une concentration de $0,01 \mathrm{mg} \cdot \mathrm{l}^{-1} \mathrm{Cd}$ $\left(Q_{10 \mathrm{ppb}}\right)$ ou de $1 \mathrm{mg} \cdot \mathrm{i}^{-1} \mathrm{Cd}\left(Q_{1000 \mathrm{ppb}}\right)$ dans la solution d'équilibre.

$\mathrm{Mg}$ ) échangeable. L'analyse prédictive du modèle est considérablement améliorée lorsqu'on utilise des régressions multiples qui prennent simultanément en considération plusieurs proprié-

Tableau III. Coefficients de corrélation simple (r) entre la capacité d'adsorption du $\mathrm{Cd}$ dans la région des faibles $\left(Q_{10 \mathrm{ppb}}\right)$ et des hautes $\left(Q_{1000 \mathrm{ppb}}\right)$ concentrations et les propriétés des sols.

\begin{tabular}{lrr}
\hline & $Q_{10 \rho p b}$ & $Q_{1000 p p b}$ \\
\hline Argile & & \\
pH (eau) & 0,71 & 0,73 \\
Matière organique & $-0,21$ & $-0,42$ \\
$\mathrm{CaCO}_{3}$ actif & 0,34 & 0,46 \\
$\mathrm{CaCO}_{3}$ total & 0,72 & 0,81 \\
$\mathrm{Fe}_{2} \mathrm{O}_{3}$ libre & 0,29 & 0,38 \\
$\mathrm{Fe}_{2} \mathrm{O}_{3}$ total & $-0,12$ & $-0,13$ \\
$\mathrm{MnO}_{2}$ total & $-0,13$ & $-0,17$ \\
$\mathrm{CEC}\left(\mathrm{AcNH} \mathrm{H}_{4}\right)$ & $-0,07$ & $-0,15$ \\
$\mathrm{CEC}\left({ }^{45} \mathrm{Ca}\right)$ & 0,65 & 0,68 \\
$(\mathrm{Ca}+\mathrm{Mg})$ éch. & 0,53 & 0,63 \\
& 0,68 & 0,72 \\
\hline
\end{tabular}

Seuil de signification pour $n=11 ; P<0,01: r=0,736$ : $P<0,05$ : $r=0.602$. tés des sols. Les relations les plus significatives sont présentées au tableau IV. II ressort de cette analyse statistique que l'utilisation simultanée de 2 variables explicatives $\left(\mathrm{CaCO}_{3}\right.$ actif et $\mathrm{CEC}$ $\mathrm{AcNH}_{4}$ ) s'avère très favorable et permet d'expliquer $70 \%\left(\mathrm{R}^{2}\right.$, coefficient de détermination multiple) de la variabilité de l'adsorption dans la région des traces et $83 \%$ dans la zone des concentrations supérieures. L'introduction de la matière organique comme troisième variable indépendante n'améliore pas significativement la valeur prédictive des équations. Ces dernières s'établissent comme suit :

$$
\begin{gathered}
\mathrm{Q}_{10 \mathrm{ppb}}=-1,93+0,77\left(\mathrm{CaCO}_{3} \text { actif }\right) \\
+0,87\left(\mathrm{CEC}\left(\mathrm{AcNH}_{4}\right)\right) \\
\mathrm{R}^{2}=0,70 \quad \\
\mathrm{Q}_{1000 \mathrm{ppb}}=30,32+23,66\left(\mathrm{CaCO}_{3} \text { actif }\right) \\
+22,92\left(\mathrm{CEC}\left(\mathrm{AcNH}_{4}\right)\right) ; \\
\mathrm{R}^{2}=0,83 \quad
\end{gathered}
$$

Comme il existe une relation linéaire entre la teneur en argile et le calcaire actif d'une part $(r=$ $0,80)$ et la $C E C\left(\mathrm{AcNH}_{4}\right)$ d'autre part $(r=0,58)$, le pourcentage d'argile, quoique directement corrélé avec l'adsorption (tableau III), n'intervient finalement pas dans l'équation de régression. 
Tableau IV. Analyse par régression multiple pas à pas reliant la capacité d'adsorption du Cd dans la région des faibles $\left(Q_{10 \mathrm{ppb}}\right)$ et des hautes $\left(Q_{1000 \mathrm{ppb}}\right)$ concentrations aux propriétés des sols.

\begin{tabular}{|c|c|c|c|c|}
\hline $\begin{array}{l}\text { Pas de la } \\
\text { régression }\end{array}$ & $Q_{10 p p b}$ & $\mathrm{R}^{2}$ & $Q_{1000 p p b}$ & $\mathrm{R}^{2}$ \\
\hline 1 & $\mathrm{CaCO}_{3}$ actif * & 0,520 & $\mathrm{CaCO}_{3}$ actif $^{*}$ & 0,657 \\
\hline 2 & CEC $\left(\mathrm{AcNH}_{4}\right)^{*}$ & 0,696 & $\mathrm{CEC}\left(\mathrm{AcNH}_{4}\right)^{*}$ & 0,832 \\
\hline 3 & Matière organique & 0,734 & Matière organique & 0,833 \\
\hline
\end{tabular}

$X=$ Variable indépendante (propriété du sol): * Variable explicative significative au seuil $P \leq 0,01$.

Les capacités d'adsorption estimées au départ des équations 1 et 2 ont été mises en relation avec les valeurs effectivement mesurées, dans le but d'illustrer la dispersion résiduelle des données qui ont servi à ajuster les paramètres (fig $3)$. On notera que la valeur prédictive de l'équation établie pour la région des traces $\left(Q_{10} \mathrm{ppb}\right)$ est sensiblement inférieure à celle établie pour les concentrations plus élevées. Ceci découle en partie de la plus grande erreur relative liée à l'estimation de $Q_{10 \mathrm{ppb}}$ (tableau II) et peut-être égale-


Fig 3. Relation entre les valeurs expérimentales de $Q_{1000}$ pop (A) ou $Q_{10}$ ppo (B) et celles estimées par régression linéaire multiple. ment, de l'absence dans l'analyse statistique d'autres variables explicatives (complexation organique, Cd préexistant dans le sol, ...).

\section{DISCUSSION}

En utilisant les valeurs des coefficients de régression partielle et les teneurs moyennes du calcaire actif et de la CEC $\left(\mathrm{AcNH}_{4}\right)$, la contribution relative du $\mathrm{CaCO}_{3}$ actif à la capacité d'adsorption de ces sols est calculée comme étant d'environ 53 et $57 \%$ pour $Q_{10}$ ppb et $Q_{1000}$ ppb, respectivement.

Le taux de calcaire actif, quoique non associé directement à la notion de calcaire fin, est un indice qui renseigne sur l'importance des surfaces externes des particules de calcite (Callot et Dupuis, 1979). Cet indice s'avère significativement corrélé avec l'adsorption, tant dans la première $(r=0,72)$ que dans la seconde $(r=0,81)$ partie de l'isotherme de Freundlich. Par contre, on note que le taux du $\mathrm{CaCO}_{3}$ équivalent total n'explique en rien la variabilité de l'adsorption observée ( $r=$ 0,29 et 0,33 pour les segments 1 et 2 , respectivement). Aux faibles concentrations en $\mathrm{Cd}$ (segment 1), la relation entre l'adsorption et la teneur en calcaire actif s'explique vraisemblablement par la chimisorption. Le maintien de la relation adsorption - $\% \mathrm{CaCO}_{3}$ actif aux concentrations plus élevées pourrait s'expliquer par la nucléation du $\mathrm{CdCO}_{3}$ - ou la formation d'une solution solide $\mathrm{Cd}_{\mathrm{x}} \mathrm{CO}_{3}-\mathrm{Ca}_{\mathrm{y}} \mathrm{CO}_{3}$ (Davis et al, 1987) à partir des surfaces de calcite couvertes en $\mathrm{Cd}^{2+}$. $\mathrm{Ce}$ résultat s'accorde également avec l'hypothèse d'un continuum entre adsorption et précipitation, tel que proposé par Comans et Middelburg (1987).

Dans les sols non calcaires, l'adsorption du Cd est avant tout déterminée par des processus 
d'échange ionique. Considérées conjointement avec le pH, la CEC ou la somme des bases échangeables apparaissent fréquemment comme de bons estimateurs de la capacité d'adsorption des sols (Singh, 1979; Mc Bride et al, 1981) ainsi que de leur faculté à prévenir l'adsorption du métal par la plante (Miller et al, 1976; Mc Bride et al, 1981; Hinesly et al, 1982). Dans le cas présent, où l'on s'adresse à une série de sols relativement uniformes du point de vue du $\mathrm{pH}$, la $\mathrm{CEC}$ - ou même la somme $\left(\mathrm{Ca}^{2+}+\mathrm{Mg}^{2+}\right)$ échangeable - n'explique qu'une part (40-50\%) de la variabilité de l'adsorption. Cette fraction n'est d'ailleurs pas différente lorsque l'on considère la $C E C\left({ }^{45} \mathrm{Ca}\right)$ plutôt que la $\mathrm{CEC}\left(\mathrm{ACNH}_{4}\right)$. $\mathrm{Ce}$ dernier résultat peut paraître étonnant puisque la $C E C\left({ }^{45} \mathrm{Ca}\right)$ est sensée traduire l'ensemble des sites où le calcium est isotopiquement échangeable, c'est-à-dire non seulement les sites d'échange - après saturation en $\mathrm{Ca}$ mais aussi les sites de surface des particules de $\mathrm{CaCO}_{3}$ (Lahav et Bolt, 1964; Van Bladel et al, 1975).

D'une façon générale, la capacité d'adsorption du cadmium des sols étudiés est particulièrement élevée. Dans une étude similaire (Halen et Van Bladel, 1990) où les isothermes d'adsorption ont été établies en milieu $\mathrm{CaCl}_{2} \quad 0,0033$ mol $\left.\right|^{-1}$, on a mesuré, pour une série de 24 sols non calcaires de Belgique, des valeurs moyennes de $Q_{10}$ ppb et $Q_{1000}$ ppb respectivement égales à $4,5 \pm 8,3 \mathrm{mg} \cdot \mathrm{kg}$ et $51 \pm 21 \mathrm{mg} / \mathrm{kg}$. Dans l'étude actuelle, l'adsorption est $3\left(Q_{10} \mathrm{ppb}\right)$ à 10 fois $\left(Q_{1000} \mathrm{ppb}\right)$ plus importante (tableau II) bien que les isothermes aient été établies à une force ionique largement supérieure. Le surcroît d'adsorption, en sol calcaire, doit être attribué non seulement à la présence de surfaces de $\mathrm{CaCO}_{3}$ à haut pouvoir de rétention, mais aussi, indirectement, aux $\mathrm{pH}$ généralement élevés de ces sols.

Il convient enfin de souligner qu'en milieu naturel, le comportement des métaux lourds dans le système sol-eau est déterminé en premier lieu par des paramètres physico-chimiques tels que la force ionique de la solution du sol et sa composition. Pour permettre l'analyse des effets des propriétés intrinsèques des sols, ces variables sont éliminées, lors de l'établissement des isothermes d'adsorption, par le recours à une solution saline $\left(\mathrm{CaCl}_{2} 0,\left.01 \mathrm{~mol} \cdot\right|^{-1}\right)$ et l'utilisation de rapports sol solution beaucoup plus faibles que ceux en plein champ.

Il est reconnu que les chlorures, fréquemment abondants dans les sols des zones arides, for- ment des complexes stables avec les ions $\mathrm{Cd}^{2+}$ (Garcia-Mirayaga et Page, 1976; Bingham et al, 1984; O'Connor et al, 1984; Palheiros et al, 1989). Ceci est illustré à la figure 4 où l'on compare, pour les sols 1 et 9 , les isothermes d'adsorption en milieu $\mathrm{CaCl}_{2}$ 0,01 molol-1 aux isothermes en milieu $\mathrm{Ca}\left(\mathrm{ClO}_{4}\right)_{2} \quad 0,01 \mathrm{~mol} \cdot \mathrm{f}^{-1}$. On considère que les ions $\left(\mathrm{ClO}_{4}-\right)$ ne forment pas de paires ioniques avec le cadmium (GarciaMiragaya et Page, 1976). En milieu $\mathrm{CaCl}_{2} \quad 0,01$ mol॰1-1 par contre, le calcul des équilibres ioniques fait apparaître que, pour les points expérimentaux des segments 1 des isothermes, $51,4 \%$ $\mathrm{du} \mathrm{Cd}$ en solution se trouve sous forme complexée ( $\mathrm{CdCl}+(48,4 \%), \mathrm{CdCl}_{2}(2,9 \%)$ et $\mathrm{CdOH}^{+}$ $(\approx 0,1 \%))$ et la fraction restante sous forme de $\mathrm{Cd}^{2+}$ libre. Cette complexation, encore plus prononcée dans la partie 2 des isothermes $(62 \%$ aux taux d'apports les plus élevés en $\mathrm{CdCl}_{2}$ ) a pour effet de diminuer l'adsorption (fig $4 \mathrm{~A}$ et $\mathrm{C}$ ). Toutefois, lorsque les quantités dans l'un et l'autre système sont exprimées en fonction des activités en $\mathrm{Cd}^{2+}$ dans la solution d'équilibre, les isothermes se révèlent identiques (fig $4 B$ et $D$ ). Ceci signifie que les paires d'ions formées en milieu chlorure sont peu ou pas compétitives vis-àvis du $\mathrm{Cd}^{2+}$, pour l'adsorption. Par ailleurs, ceci montre également que les effets de la complexa-

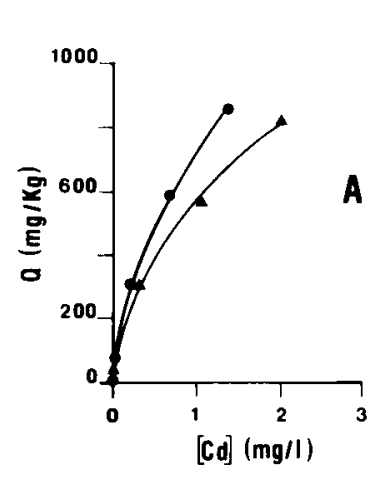

Sol 1

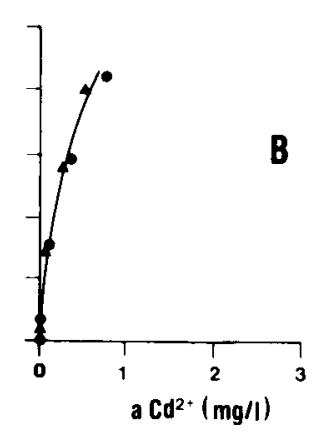

Sol 9
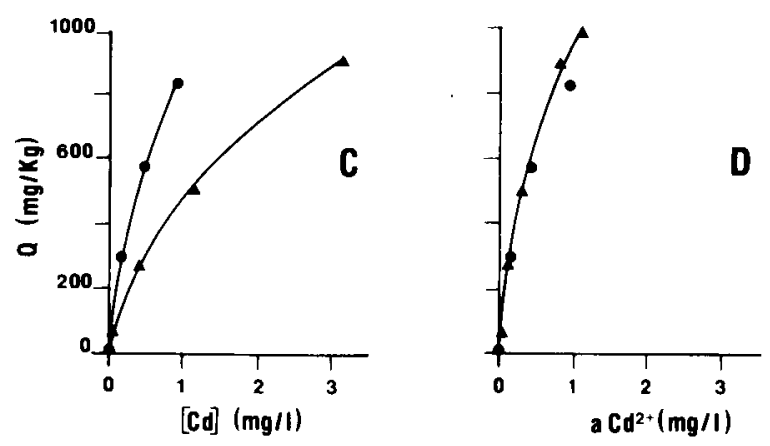

Fig 4. Adsorption du cadmium par les sols $1(A, B)$ et 9 (C, D) en fonction de la concentration totale en $C d(A, C)$ et en fonction de l'activité en $\mathrm{Cd}^{2+}(\mathrm{B}, \mathrm{D})$ dans la solution d'équilibre, en milieu $\mathrm{Ca}\left(\mathrm{ClO}_{4}\right)_{2} 0,01 \mathrm{~mol}^{-1}(\mathrm{O})$ et $\mathrm{CaCl}_{2} \quad 0,01$ $\mathrm{mol} \cdot \mathrm{I}^{-1}(\Delta)$. 
tion du cadmium - de même que, théoriquement, ceux de la force ionique (O'Connor et al, 1984; Hirsh et al, 1989) - peuvent être raisonnablement prédits à partir d'un calcul des activités des espèces chimiques en solution. Les équations de Freundlich peuvent dès lors être exprimées en termes d'activités (tableau V), ce qui leur donne une portée plus large. Redéfinissant le paramètre $Q_{10}$ ppb comme la quantité de Cd adsorbée pour une activité de $8,8.10^{-8} \mathrm{~mol} \cdot \mathrm{l}^{-1}$ (soit $0,01 \mathrm{mg} / \mathrm{l}$ $\mathrm{Cd}^{2+}$ ) dans la solution d'équilibre, la nouvelle équation prédictive s'établit comme suit :

$$
\begin{aligned}
\mathrm{Q}_{10 \mathrm{ppb}}= & -10,91+2,90\left(\mathrm{CaCO}_{3} \text { actif }\right) \\
& +2,96\left(\mathrm{CEC}\left(\mathrm{AcNH}_{4}\right)\right) \\
\mathrm{R}^{2}=0,70 &
\end{aligned}
$$

\section{CONCLUSION}

En comparaison des sols non calcaires, où la capacité de rétention du cadmium est avant tout déterminée par le $\mathrm{pH}$ et les propriétés d'échange de sols (CEC, $\left(\mathrm{Ca}^{2+}+\mathrm{Mg}^{2+}\right)$ échangeables), les sols calcaires font preuve d'un pouvoir d'adsorption particulièrement élevé. Ce surcroît d'adsorption est attribué à la forte réactivité des surfaces de calcite, expliquée en termes de chimisorption aux faibles taux d'apports en Cd et en termes de nucléation - précipitation aux taux d'apports plus élevés. Le paramètre calcaire actif semble être un bon estimateur de la plus ou moins grande réactivité du $\mathrm{CaCO}_{3}$ vis-à-vis du $\mathrm{Cd}$, alors que la teneur totale en équivalent de $\mathrm{CaCO}_{3}$ n'explique

Tableau V. Equations de Freundlich $\left(Q=K A^{n}\right)$ pour l'adsorption du cadmium dans la région des traces par 12 sols calcaires de la région du Sud-Est de l'Espagne ( $A$ activité du $\mathrm{Cd}^{2+}$, est exprimé en $\mathrm{mg}^{\cdot 1-1} ; \mathrm{Q}$, quantité de Cd adsorbée, en $\mathrm{mg} \cdot \mathrm{kg}^{-1}$ ).

Sol

\begin{tabular}{rrr}
\hline 1 & & \\
2 & 2455 & 0,86 \\
3 & 3507 & 0,90 \\
4 & 1662 & 0,84 \\
5 & 3442 & 0,87 \\
6 & 531 & 0,79 \\
7 & 1447 & 0,80 \\
8 & 9202 & 0,91 \\
9 & 667 & 0,81 \\
10 & 2221 & 0,87 \\
11 & 822 & 0,86 \\
12 & 389 & 0,73 \\
\hline
\end{tabular}

généralement pas la variabilité de l'adsorption. Les capacités d'adsorption relatives des sols de la zone étudiée peuvent être valablement estimées au départ des valeurs de $\operatorname{CEC}\left(\mathrm{AcNH}_{4}\right)$ et de teneurs en $\mathrm{CaCO}_{3}$ actif.

\section{REMERCIEMENTS}

Les auteurs remercient vivement Mme $N$ Devilez et M.A Vandenbosch pour leur précieuse collaboration technique. Ce travail s'inscrit dans le cadre des recherches subventionnées par l'Institut pour l'encouragement de la recherche scientifique dans l'industrie et l'agriculture (IRSIA), Belgique.

\section{RÉFÉRENCES}

Allison FE, Moodie OD (1965) Carbonate. In: Methods of soil analyses, part 2 (CA Black et al, eds). Agronomy 9, Am Soc Agron, Madison, Wisconsin, 1379-1396

Alloway BJ (1990) Cadmium. In: Heavy metals in soils (Alloway BJ, ed), Blackie \& son Ltd, $339 p$

Alloway BJ, Thornton I, Smart GA, Sherlock JC, Quinn MJ (1988) Metal availability. Sci Total Environ $75,41-69$

Ameryckx J, Verheye W (1984) Fractions minérales et classification de la texture du sol. Pédologie 34, 319-330

Bingham FT, Sposito G, Strong JE (1984) The effect of chloride on the availability of cadmium. J Environ Qual 13, 71-74

Bonneau M, Souchier B (1979) Pédologie. 2. Constituants et propriétés du sol. Masson, Paris, $459 p$

Callot $G$, Dupuis M (1979) Le calcaire actif des sols et sa signification. Sci Sol Bull AFES 198, 17-26

Cavallaro N, Mc Bride MB (1978) Copper and cadmium adsorption characteristics of selected acid and calcareous soils. Soil Sci Soc Am J 42, 550556

Comans RNJ, Middelburg JJ (1987) Sorption of trace metals on calcite: applicability of the surface precipitation model. Geochim Cosmochim Acta 51, 2587-2591

Davis JA, Fuller CC, Cook AD (1987) A model for trace metal sorption process at the calcite surface : adsorption of $\mathrm{Cd}^{2+}$ and subsequent solid solution formation. Geochim Cosmochim Acta 51, 14771490

Estañ MT, Bolarin MC, Guillen MG (1987) Adsorcion de cadmio en suelos calizos. Estimacion de parametros de diversas isotermas por metodos de regresion no lineal. Agrochimica 31, 500-509

Friberg L, Piscator M, Nordberg G, Kjellstrom T (1974) Cadmium in the environment, CRC Press, Cleveland, Ohio, Second edn, $248 p$ 
Garcia-Miragaya J, Page AL (1976) Influence of ionic strength and inorganic complex formation on the sorption of trace amounts of cadmium by Montmorillonite. Soil Sci Soc Am J 40, 658-663

Garcia-Miragaya J, Page AL (1978) Sorption of trace quantities of cadmium by soils with different chemical and mineralogical composition. Water Air Soil Pollut 9, 289-299

Gardiner J (1974) The chemistry of cadmium in natural water. I. A study of cadmium complex formation using the cadmium specific ion electrode. Water Res 8, 23-30

Gerritse R, Van Driel W, Smilde K, Van Luit B (1983) Uptake of heavy metals by crops in relation to their concentration in the soil solution. Plant Soil 75 , 393-404

Halen H, Van Bladel R (1990) Etude des facteurs contrôlant l'adsorption du cadmium dans une série de sols belges. Rev Agric 43, (sous presse)

Hinesly TD, Redborg KE, Ziegler EL, Alexander JD (1982) Effect of soil cation exchange capacity on the uptake of cadmium by corn. Soil Sci Soc Am J $46,490-497$

Hirsch D, Nir S, Banin A (1989) Prediction of cadmium complexation in solution and adsorption to Montmorillonite. Soil Sci Soc Am J 53, 716-721

Jarvis SC, Jones LHP (1980) The contents and sorption of cadmium in some agricultural soils of England and Wales. J Soil Sci 31, 469-479

King LD (1988) Retention of cadmium by several soils of the southeastern United States. J Environ Qual $17,246-250$

Kjeilstrom T, Nordberg GF (1978) A kinetic model of cadmium metabolism in human beings. Environ Res 16, 248-263

Lahav N, Bolt GH (1964) Self diffusion of ${ }^{45} \mathrm{Ca}$ into carbonates. Soil Sci 97, 293-299

Leeper GW (1952) Factors affecting availability of inorganic nutrients in soils with special reference to micronutrient metals. Annu Rev Plant Physio/ 3, 1

Levi-Minzi R, Soldatini GF, Riffaldi R (1976) Cadmium adsorption by soils. $J$ Soil Sci $27,10-15$

Mahler RJ, Bingham FT, Sposito G, Page AL (1980) Cadmium enriched sewage sludge application to acid and calcareous soils: Relation between treatment, cadmium in saturation extracts, and cadmium uptake. J Environ Qual 9, 359-363

Mc Bride MB (1980) Chemisorption of $\mathrm{Cd}^{2+}$ on calcite surfaces. Soil Sci Soc Am J 44, 26-28

Mc Bride MB, Tyler LD, Hovde DA (1981) Cadmium adsorption by soils and uptake by plants as affec- ted by soil chemical properties. Soil Sci Soc Am J 45, 739-744

Miller JE, Hassett JJ, Koeppe DE (1976) Uptake of cadmium by soybeans as influenced by soil cation exchange capacity, $\mathrm{pH}$, and available phosphorus. J Environ Qual 5, 157-160

Navrot J, Singer A, Banin A (1978) Adsorption of cadmium and its exchange characteristics in some Israeli soils. J Soil Sci 29, 505-511

O'Connor GA, O'Connor C, Cline GR (1984) Sorption of cadmium by calcareous soils: Influence of solution composition. Soil Sci Soc Am J 48, 1244-1247

Palheiros IB, Costa Duarte A, Oliveira JP, Hall A (1989) The influence of $\mathrm{pH}$, ionic strength and chloride concentration on the adsorption of cadmium by a sediment. Water Sci Technol 21, 1873-1876

Papadopoulos P, Rowell DL (1988) The reactions of cadmium with calcium carbonate surfaces. J Soil Sci 39, 23-26

Roels H, Lauwerys RR, Buchet JP, Bernard A (1981) Environmental exposure to cadmium and renal function of aged women in three areas of Belgium. Environ Res 24, 117-130

Ryan JA, Pahren HR, Lucas JB (1982) Controlling cadmium in the human food chain: a review and rationale based on health effects. Environ Res 28, 251-302

Singh S (1979) Sorption and release of cadmium in some Canadian soils. Can J Soil Sci 59, 119-136

Soon YK (1981) Solubility and sorption of cadmium in soils amended with sewage sludge. J Soil Sci 32 , 85-95

Street JJ, Lindsay WL, Sabey BR (1977) Solubility and plant uptake of cadmium in soils amended with cadmium and sewage sludge. J Environ Qual 6, 72-77

Van Bladel R, Menzel R (1969) A thermodynamic study of sodium-strontium exchange on Wyoming Bentonite. Proc Int Clay Conf (Tokyo) 1, 619-634

Van Bladel R, Frankart R, Gheyi HR (1975) A comparison of three methods of determining the cation exchange capacity of calcareous soils. Geoderma 13, 289-298

Verloo M, Willaert G, Eeckout M, De Spiegeleer F (1987) Chemical and biological implications of metal speciation in contaminated soils. In: Proc Int Conf Heavy Metals in the Environment, vol 1, New Orleans, sept 87, 253-255

Wagman DD, Evans WH, Parker VB, Halow I, Bailey SM, Schumm RH (1968) Selected values of chemical thermodynamic properties. NBS Tech Note 270.3 US Dep of Commerce, Nat Bur of Stand, Washington, DC 